\section{Adaptaciones metodológicas en la aplicación del análisis estratigráfico constructivo: el Santuario de San Juan de la Penyagolosa (Castellón) y la iglesia de San Juan de los Reyes en Granada}

Camilla Mileto, Fernando Vegas

Universidad Politécnica de Valencia

\begin{abstract}
Resumen
En este texto se presentan dos casos de aplicación del estudio estratigráfico constructivo en los cuales se desarrollaron dos adaptaciones específicas ocasionadas por el caso concreto del edificio analizado. En el primer caso, el estudio del Santuario de San Juan de la Peñagolosa, la notable dimensión del conjunto conllevaba una complejidad de recogida y gestión de la información. Por esta razón, se ensayó una metodología de jerarquización de la información que permitiera una gestión de la misma más rápida y eficaz. En el segundo caso, el estudio de la iglesia de San Juan de los Reyes, además del estudio estratigráfico se realizó un estudio complementario. La misma técnica utilizada en el edificio (fábricas de machones de ladrillo y cajones de cantos rodados y cal) sugirió la posibilidad de realizar el estudio del ritmo de la distribución de los mechinales.
\end{abstract}

Palabras clave: estratigrafía, arquitectura, metodología, jerarquización de la información, mechinales

\begin{abstract}
This text presents two cases of the application of stratigraphic wall study in which two specific adaptations, stemming from the original case of the building analysed, were developed. In the first case, the study of the Santuario de San Juan de la Peñagolosa, the notable size of the shrine meant that data collection and management were highly complex. For this reason, a methodology was tested whereby information was hierarchized, allowing it to be managed faster and more efficiently. In the second case, the study of the church of San Juan de los Reyes, a complementary study was carried out in addition to the stratigraphic wall study. The same construction technique used in the building (wall stonework with brick buttresses and limestone boulder caissons) brought about the possibility of carrying out a study of the distribution of the weep holes.
\end{abstract}

Key words: Stratigraphy, architecture, methodology, hierarchization of information, weep holes

\section{ESTUDIO ESTRATIGRÁFICO CONSTRUCTIVO DE UN GRAN CONJUNTO HISTÓRICO: EL SANTUARIO DE SAN JUAN DE LA PENYAGOLOSA (CASTELLÓN) ${ }^{1}$}

El análisis estratigráfico constructivo realizado en el Santuario de San Juan de la Penyagolosa ha sido redactado como parte de un estudio previo más amplio. El objetivo general del encargo, propuesto por la Generalitat Valenciana, era el estudio pormenorizado del conjunto y la documentación del estado existente, principalmente, en sus caracteres materiales, técnicas constructivas y materiales utilizados, y en su evolución constructiva en el tiempo, en aras de proporcionar un conocimiento detallado del conjunto existente para, en caso de necesidad, poder comprobar eventuales cambios o intervenciones realizadas en los últimos años. Además, se solicitó la elaboración de algunas notas en relación con los posibles fenómenos de deterioro y de daño estructural, para poder encargar eventualmente un proyecto de restauración.

La estratigrafía constructiva es, por tanto, el medio utilizado no sólo para realizar el estudio de las fases constructivas del edificio, sino también para documentar detalladamente todos los elementos, materiales y técnicas constructivas presentes en el conjunto en su estado actual. Se puede afirmar por ello que en este estudio el levantamiento estratigráfico tuvo tres vertientes: en primer lugar, la documentación del patrimonio existente en sus componentes materiales; en segundo lugar, la base para la redacción de las hipótesis de las fases constructivas; $y$, en tercer lugar, el enlace con el eventual proyecto de restauración.

\subsection{El Santuario de San Juan de la Penyagolosa}

El Santuario, que se ubica en el término municipal de Vistabella del Maestrazgo en la provincia de Castellón, está formado por un conjunto de edificios dispuestos en torno a una plaza de acceso general y a un pequeño patio interior que articula los accesos a los diferentes cuerpos de fábrica. El Santuario, posiblemente construido en su parte más antigua en el siglo XVI sobre una ermita existente, ha sufrido una serie de ampliaciones y modificaciones en los siglos sucesivos. Actualmente, el conjunto se compone de una iglesia, un cuerpo central en torno al patio, un edificio residencial, y dos edificios separados del resto, una construcción adaptada en la actualidad para barbacoas y un cuerpo anexo.

${ }^{1}$ El análisis estratigráfico del Santuario de San Juan de la Penyagolosa ha sido realizado por los autores en el ámbito de un estudio previo más amplio, realizado por un grupo de investigadores dirigido por los arquitectos Juan Francisco Noguera Giménez y Fernando Vegas López-Manzanares. El trabajo se ha realizado por encargo de la Consellería de Cultura y Ciencia de la Generalitat Valenciana, en el año 2000. La infografía de los planos de levantamiento estratigráfico y de las hipótesis de períodos constructivos ha sido realizada por M. ${ }^{a}$ José Miñarro Viseras y Ángela López Sabater 


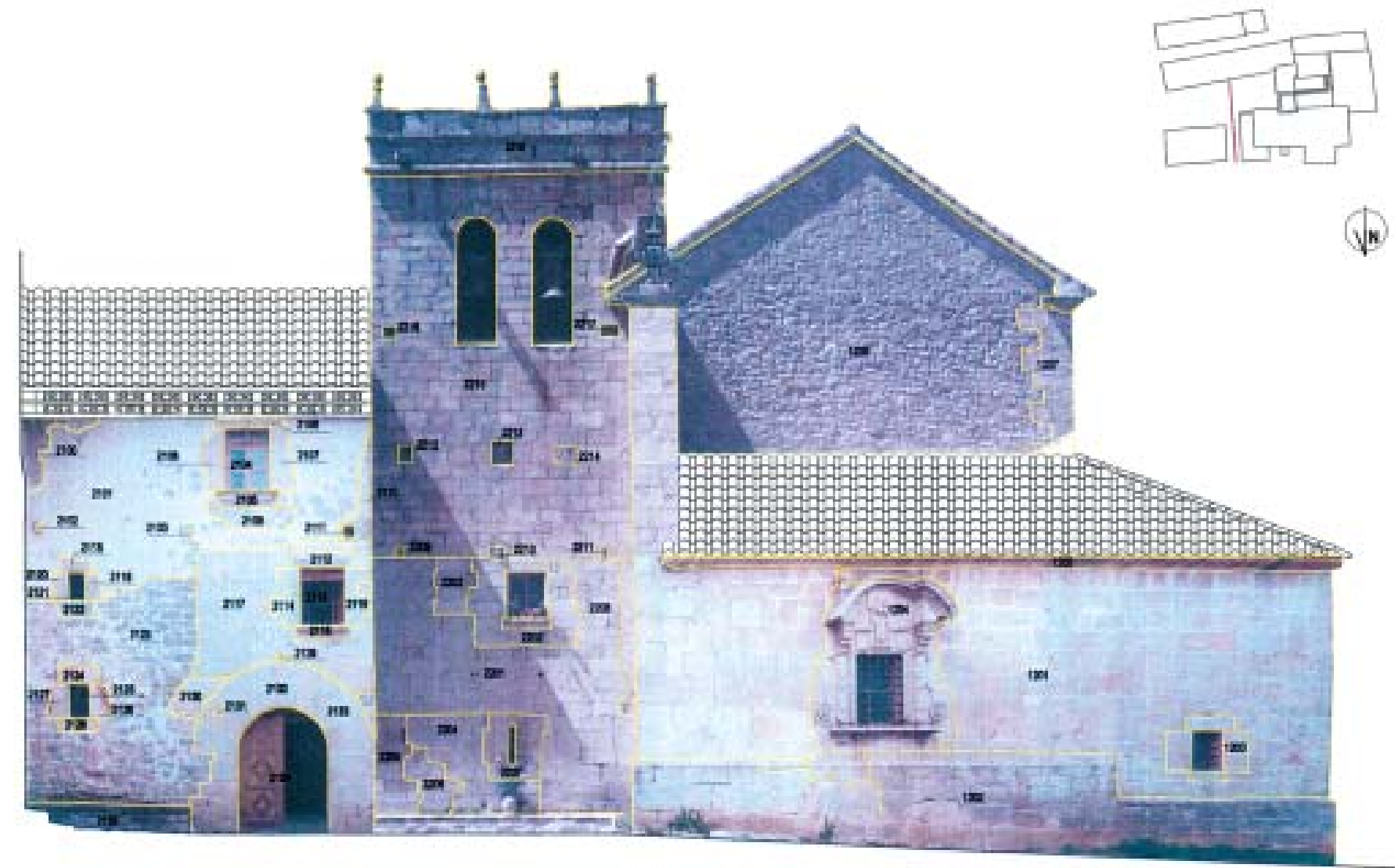

Fig. 1. Plano de análisis estratigráfico del alzado Este del Santuario de San Juan de la Peñagolosa (Castellón)

La iglesia posee una sola nave y cuatro tramos. Los tres primeros están cubiertos de bóvedas con lunetos y el cuarto con una cúpula sobre pechinas. El coro se sitúa en el primer tramo, a los pies de la nave. El edificio central constituye el núcleo del conjunto y está organizado alrededor de un patio rectangular central que funciona como rótula de todo el conjunto, ya que a su alrededor se organizan una serie de entradas y escaleras de acceso a los demás cuerpos. La gran ala del conjunto, ubicada en la parte oriental, es un edificio destinado en su totalidad a residencia, tanto en la planta baja, porticada, con una serie de pequeñas habitaciones separadas, como en la planta superior organizada a partir de un largo pasillo que distribuye a las habitaciones. Por último, las dos construcciones separadas del conjunto consisten en un edificio con espacio único y cubierta de cerchas de madera, destinado a alojar comidas de barbacoa y ubicado en un lado de la plaza de acceso, y un edificio ubicado a la espalda del conjunto en el lado meridional, destinado a servicios y habitaciones.

\subsection{La metodología adoptada (Fig. 1 y 2)}

El análisis estratigráfico se realizó en la totalidad del Santuario, un extenso conjunto formado por una serie de edifi- cios y dependencias construidas en tiempos diferentes. Por la notable dimensión del conjunto, la recogida de la información estratigráfica se presentaba onerosa y compleja. En aras a facilitar la gestión de una gran cantidad de información, se siguió una metodología que previó una recogida de la información según diferentes niveles de aproximación, desde el más general hacia el más detallado ${ }^{2}$.

En una primera fase, se ha estudiado el santuario como conjunto de cuerpos de fábrica, diferentes pero adosados los unos a los otros, cuyas relaciones, de contemporaneidad o anterioridad/posterioridad, se han podido leer en los puntos de contacto entre los muros, según un método más propio del levantamiento crítico-constructivo, tanto en la planta como en los alzados generales del conjunto. En una segunda fase, se han estudiado los cuerpos de fábrica singularmente, documentando para cada uno de ellos los alzados de detalle, hasta llegar a la definición de las unidades estratigráficas constructivas.

\footnotetext{
${ }^{2}$ En este sentido se podría afirmar que se siguió, al menos en algunos aspectos, la propuesta de Brogiolo, que consiste en la recogida de la información por niveles diferentes de detalle. Cfr: Brogiolo G. P., 1988, $A r-$ cheologia dell'edilizia storica, Como.
} 

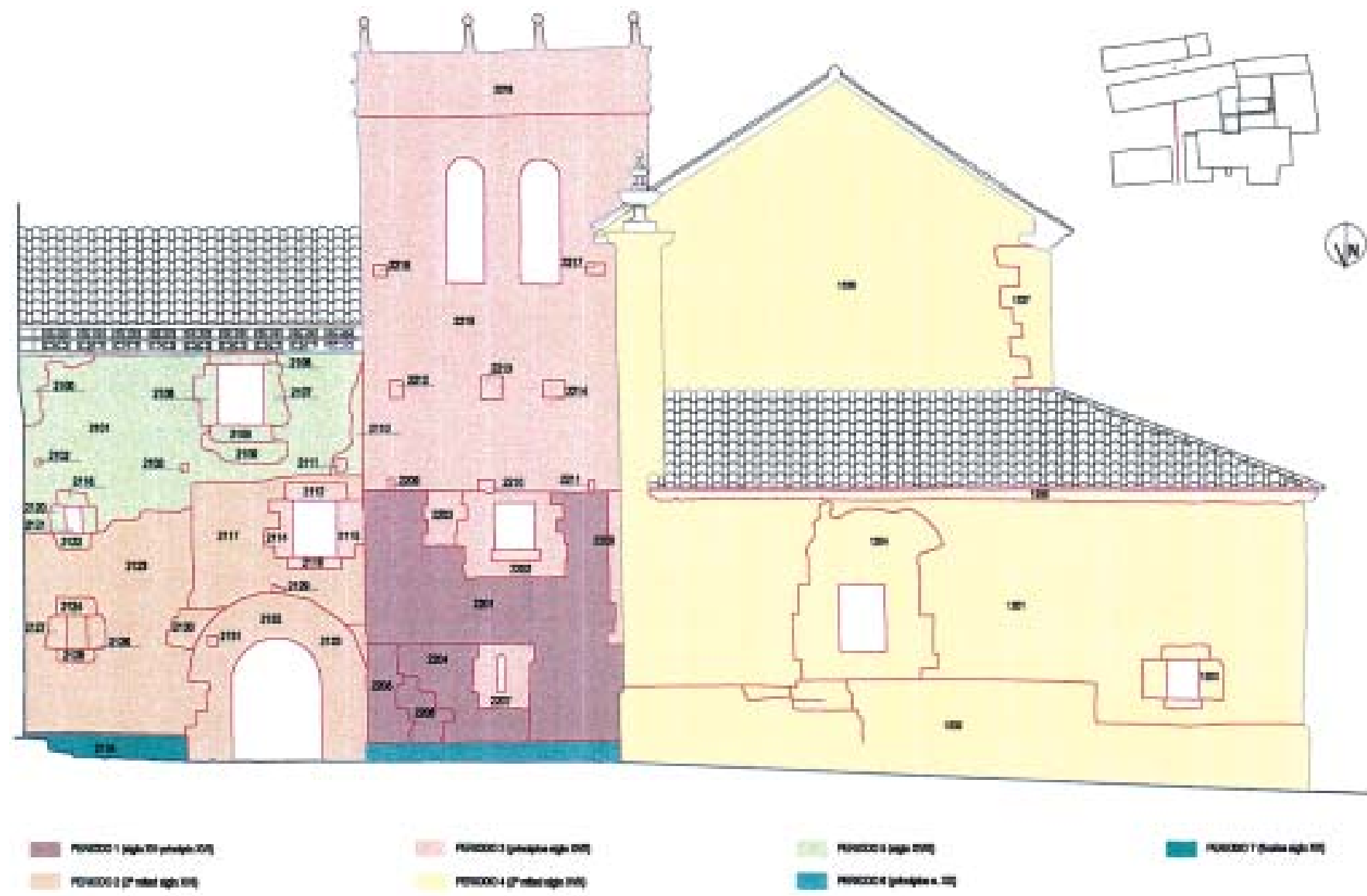

Fig. 2. Plano de hipótesis de periodos constructivos del alzado Este del Santuario de San Juan de la Peñagolosa (Castellón)

Aunque la realización del estudio se ha caracterizado por la recogida de la documentación en diferentes niveles de aproximación (desde lo general hasta lo particular), todo el conjunto ha sido estudiado con el nivel de detalle aportado por el estudio de las unidades estratigráficas murarias. Los niveles de aproximación a la información se han utilizado simplemente como forma de ordenación y jerarquización de los datos que, de otra manera, habrían representado una cantidad imposible de gestionar.

\subsection{Descripción de la hipótesis de los periodos constructivos del santuario}

Se han identificado substancialmente siete periodos constructivos y se ha elaborado una hipótesis de evolución. Es necesario precisar que se trata de una periodización realizada basándose en los datos obtenibles actualmente y que, por tanto, se trata de una hipótesis que eventualmente una nueva investigación podría confirmar o modificar.

\section{Periodo 1}

Se trata del periodo (entre el siglo XV y la primera mitad del siglo XVI) identificable con algunos restos de fábrica no siempre referible a una organización de cuerpos construidos de clara identificación: una serie de edificaciones que no permiten reconstruir una hipótesis de conjunto edificado coherente. Se trata de dos paredes situadas en el patio central, que presentan dos esquinas de sillares y paramento de mampostería.

A este mismo periodo se podría asignar el cuerpo de la gran chimenea con el gran arco de entrada, hoy en parte tapiado, que tenía que abrirse en un espacio de acogida, como se puede todavía ver en el Real Santuario de la Virgen de la Salud de Traiguera. La dimensión de esta misma chimenea permite pensar que el edificio, probablemente una primitiva hospedería, debía acoger, ya en esos tiempos, una gran cantidad de personas.

\section{Periodo 2}

Corresponde con la segunda mitad del siglo XVI, de acuerdo con la fecha encontrada en un rótulo de 1567 en el interior de una de las estancias de la planta primera del cuerpo de fábrica, actualmente utilizado como restaurante. Este cuerpo parece adosarse al cuerpo anterior de la chimenea y consta de dos plantas: planta baja constituida por dos gran- 
des salones, de los cuales uno está formado por tres grandes arcos de medio punto de sillería. La planta superior está constituida por un gran salón con dos ventanas con poyos incorporados que volcaban al patio interior, y una ventana del mismo tipo que recaía a la parte posterior del santuario. Gran parte del patio actual también debía formar parte de este conjunto y desde éste se debía acceder a una primera planta que probablemente se extendía hasta ocupar el espacio de la actual torre.

\section{Periodo 3}

De este tercer periodo encontramos una fecha, 1602, en una ventana del cuerpo añadido situado en la parte occidental del patio. La construcción de este cuerpo supuso probablemente la ampliación o modificación del lado occidental del patio. Probablemente se trata también del periodo en que se construye la actual torre.

\section{Periodo 4}

Se trata del momento en que se construye la iglesia, que presenta en la portada la fecha de 1706 . Siendo la portada probablemente lo último que se añade al edificio se supone que la iglesia haya sido construida entre finales del siglo XVII y el 1706. Es posible también que la fase constructiva correspondiente al inicio de la construcción de la iglesia enlace directamente con la fase correspondiente a la construcción de la torre.

\section{Periodo 5}

Es el periodo correspondiente a la anexión de las dependencias de habitaciones para los peregrinos, probablemente a lo largo del siglo XVIII, a continuación de la construcción de la iglesia, para poder albergar un mayor número de personas. Se trata de la construcción de los tres edificios ubicados en la zona más oriental del conjunto: el primero es el edificio de gran longitud que conforma la plaza delantera del conjunto, caracterizado por las arcadas en el alzado norte; el segundo consiste en el otro edificio que conforma la plaza y que ha sido reconstruido en un periodo muy reciente, debido a que se encontraba en un estado ruinoso; el tercer edificio construido en este periodo es el cuerpo que se encuentra exento en la parte trasera del ala con arcadas.

\section{Periodo 6}

Al principio del siglo XX corresponde, al menos en lo que se puede ver en este momento, sólo la decoración de uno de los salones de la primera planta: en el cuerpo occidental que daba al patio y que había sido construido probablemente en 1602, se inserta una pared para separar el salón del pasaje que se crea hacia el patio.

\section{Periodo 7}

Corresponde a un tiempo muy reciente que ha visto en los últimos veinte o treinta años una serie de obras para la rehabilitación del conjunto que se encontraba hasta este momento en estado de abandono. Se trata principalmente de obras de adaptación de los edificios al uso como albergue y restaurante, reparación de cubiertas y remodelación de algunos espacios interiores.

\section{EL ESQUEMA DE DISTRIBUCIÓN DE LOS MECHINALES COMO AYUDA PARA LA DATACIÓN DE LAS FASES CONSTRUCTIVAS DE UN EDIFICIO: LA IGLESIA DE SAN JUAN DE LOS REYES EN GRANADA ${ }^{3}$}

El análisis estratigráfico de la iglesia de San Juan de los Reyes ha sido realizado en el ámbito de un proyecto de restauración cuya obra ya se estaba ejecutando en el momento del estudio. Con el estudio se pretendía, por una parte, documentar los datos materiales que estaban emergiendo durante las intervenciones y, por otra parte, obtener una serie de sugerencias para la ejecución de la obra sobre un edificio que estaba revelando más riqueza de datos de los que se había previsto antes de empezar la intervención.

El estudio debía, por tanto, documentar el mayor número posible de datos legibles en el estado en el cual se encontraba el edificio, ya en fase avanzada de obra y, al mismo tiempo, señalar una serie de datos interesantes para la prosecución de la obra en el respeto de los nuevos datos aparecidos.

\subsection{La iglesia de San Juan de los Reyes en Granada}

La iglesia de San Juan de los Reyes se ubica en la Calle de los Reyes en el barrio del Albaicín de Granada, exactamente en frente de la Alhambra, en la vertiente opuesta respecto al río Darro. Se trata de una iglesia a planta rectangular de tres naves, divididas por pilastras compuestas que soportan arcos ligeramente apuntados, con un presbiterio poligonal en la cabecera de la nave principal. En el lado septentrional del presbiterio se ubica, adyacente a la nave, la torre campanario, mientras que, en posición simétrica en el lado meridional, se sitúa la sacristía. La iglesia posee dos entradas, una ubicada en el lado occidental, más antigua, y una en el lado sur, abierta hacia la Calle de los Reyes, más reciente.

\footnotetext{
3 El análisis estratigráfico de la iglesia de San Juan de los Reyes ha sido desarrollado, durante el año 2000, por los autores a solicitud de los arquitectos Gabriel Fernández Adarve, Ignacio Vélez Fernández y Antonio Martín Muñoz encargados de la restauración de la iglesia, por la Consejería de Cultura de Andalucía. La infografía de los planos de levantamiento estratigráfico y de las hipótesis de periodos constructivos ha sido realizada por M. ${ }^{a}$ José Miñarro Viseras
} 
Fig. 3. Plano de análisis estratigráfico del alzado Oeste interior de la iglesia de San Juan de los Reyes (Granada)

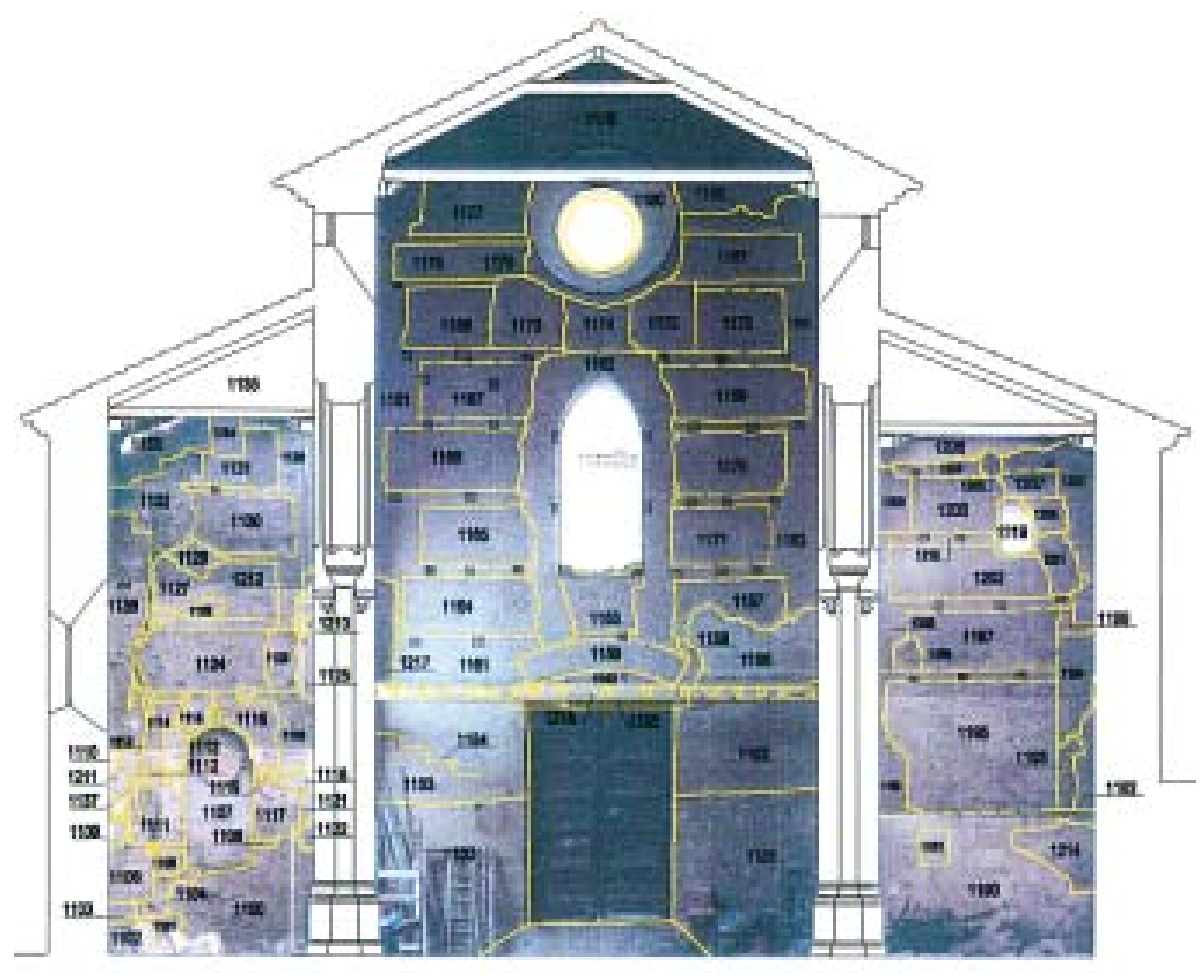

El sistema constructivo de la iglesia se caracteriza principalmente por una fábrica de cajones de cantos rodados y cal entre machones de ladrillo. Cada nivel de cajones está separado del siguiente por tres hiladas de ladrillo. La cubierta es de pares y nudillos en la nave central y consta de un alfarje inclinado en las dos naves laterales.

El edificio fue construido en 1520 sobre el solar de una antigua mezquita, de la que permanece el alminar, y luego rematado por un cuerpo añadido en su adaptación a torre campanario. La iglesia sufrió una primera intervención, que consistió solamente en la remodelación de la fachada sur, documentada en 1663. Existe documentación sobre una segunda fase de intervención en el edificio que se remonta a 1880-81. Fue posteriormente Leopoldo Torres Balbás quien intervino con una serie de reparaciones en 1929, seguido en los años cincuenta y sesenta por Francisco Prieto-Moreno. De este modo, la iglesia se presenta como un único cuerpo de fábrica estratificado a causa de las intervenciones tanto de remodelación como de restauración y reparación que se han sucedido en el transcurso del tiempo.

\subsection{La metodología adoptada}

Como se ha visto, la fábrica estudiada atesora, a pesar de presentarse como un único cuerpo compacto, una notable estratificación de intervenciones de diferente género, tanto en lo que respecta a los elementos constructivos como en lo que atañe a las superficies de acabado. El estudio se dirige por esta razón a los paramentos del edificio, tanto internos como externos, y a la identificación de las relaciones que existen entre las diversas partes heterogéneas reconocidas. De esta guisa, se han estudiado los alzados de detalle de cada una de las paredes del edificio en los cuales se han identificado las unidades estratigráficas constructivas.

La posibilidad de leer las dos caras del muro contemporáneamente permite una comparación continua que lleva a constatar, en algunos casos, la falta de correspondencia entre las dos caras, circunstancia que permite pensar en intervenciones de reparación en los paramentos.

Sin embargo, tratándose fundamentalmente de intervenciones de remodelación de las fachadas, apertura o cierre de huecos y reparaciones de vario tipo, no se ha considerado imprescindible realizar una planta con las relaciones entre las masas murarias, ya que ésta no habría podido expresar la riqueza de los datos existentes en los paramentos el edificio.

\subsection{El esquema de distribución de los mechinales} (Fig. 3 y 4 )

En el caso de la iglesia de San Juan de los Reyes ha parecido interesante realizar un estudio específico del ritmo y de la distribución de los mechinales en los paramentos en aras del establecimiento de una posible relación entre ritmos y dimensiones de éstos y posibles fases constructivas. Estos huecos, dejados por los maderos que se utilizaban 

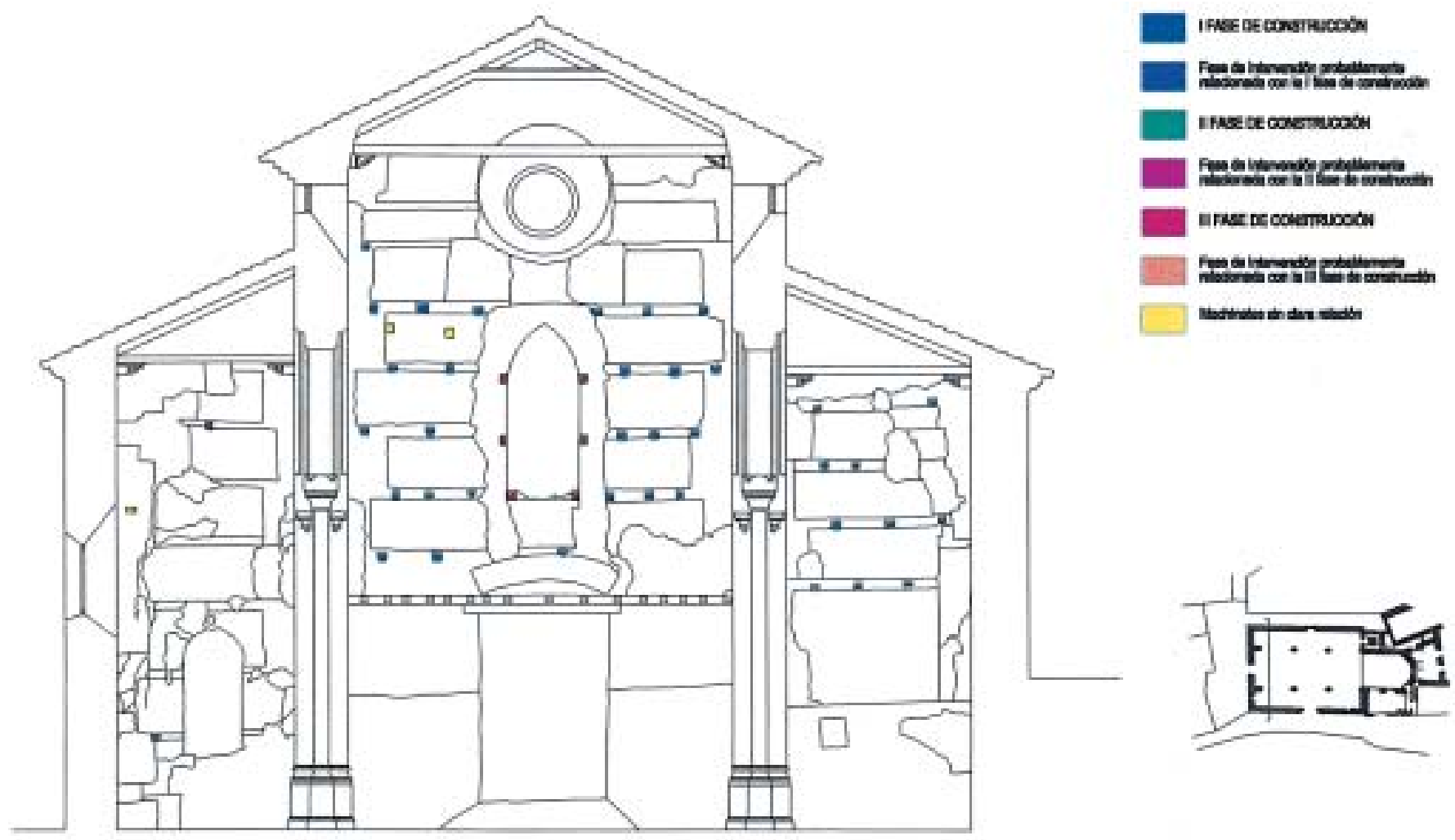

Fig. 4. Esquema de distribución de los mechinales del alzado Oeste interior de la iglesia de San Juan de los Reyes (Granada)

como andamio en la obra y que, en general, se caracterizan por una forma sin bordes de rotura, son normalmente bastante reconocibles y, por la misma razón, fácilmente documentables. Es evidente que el tamaño de los maderos utilizados, y por ende de los mechinales, y la distancia entre ellos, no tienen porqué ser constantes entre una fase y la otra de la construcción del monumento. Esta posible diversidad de dimensiones y ritmos ha llevado a realizar un estudio específico aplicando una metodología cronotipológica.

En realidad, este tipo de estudio no se ha podido extender a la totalidad de los paramentos desde el momento en que no en todos ellos ha sido posible identificar una regla, un orden o una mínima relación entre los huecos. En muchos casos, se pueden identificar huecos, posiblemente mechinales, diferentes y distribuidos de manera variada, pero las sucesivas intervenciones, y la presencia de restos de enlucidos, no permiten encontrar otros datos relacionables con ellos.

Sin embargo, en algunos paramentos, especialmente en los casos de la pared sur, interior y exterior, y del interior de la pared oeste, se ha podido extraer del ritmo de los mechinales una ley de generación que ha permitido confirmar las hipótesis de fases constructivas que se habían elaborado a través del análisis estratigráfico.
A lo largo del análisis estratigráfico (representado en los planos de análisis estratigráfico), la notable abundancia de huecos susceptibles de haber desempeñado una función de mechinal ha aconsejado no numerarlos, ni describirlos en las fichas como unidades estratigráficas, pareciendo esta operación redundante. Se ha preferido utilizar una simple cruz como sistema gráfico de representación de los mismos.

En los esquemas realizados se identifican: mechinales correspondientes a las fases de construcción, en concreto, a la primera a la segunda y a la tercera; mechinales que se pueden relacionar con los mechinales de las fases constructivas; y mechinales que no tienen un clara relación con los demás.

En el alzado oeste interior se identifica claramente la zona central del paramento de la nave principal, caracterizado por un grupo de huecos muy ordenados pertenecientes presumiblemente a la primera fase de construcción de la misma manera que, en el paramento de la nave lateral norte, se puede reconocer un grupo de huecos correspondientes a la misma fase de construcción. Alrededor del gran ventanal del paramento de la nave central, se reconocen claramente los huecos relacionados con la apertura del mismo, perteneciente a la tercera fase de construcción de la iglesia. En la nave lateral sur, se pueden identificar dos huecos relacionables con la primera fase de construcción, pero la presencia de estratos de enlucidos y las numerosas inter- 
venciones sucesivas no permiten encontrar otros mechinales para comprobarlo.

En el alzado meridional exterior en la parte de poniente de la fachada, se identifica un grupo de mechinales que por su orden, conformación y dimensión $(11,517 \mathrm{~cm}$.), se remontan a la primera fase constructiva. Por otra parte, en la zona de levante de la fachada, se reconocen dos grupos de huecos: el primero, en la parte inferior, probablemente perteneciente a la segunda fase constructiva (con una dimensión de $14 \quad 14 \mathrm{~cm}$.); el segundo, en la parte superior, por dimensión y forma se puede remitir, a su vez, a la segunda fase, aunque varíe parcialmente la disposición de los huecos. En la parte central de la fachada se encuentran, dos huecos fácilmente relacionables con la introducción de la nueva portada de piedra, en la tercera fase constructiva.

En el alzado sur interior de la misma manera que en la fachada exterior, se pueden reconocer, en la parte de poniente, un grupo de mechinales pertenecientes a la primera fase de construcción y, en la parte de levante, un grupo perteneciente a la segunda fase, además de algunos huecos correspondientes respectivamente a los dos grupos. En la parte central, es claramente identificable el grupo de huecos relacionados con la tercera fase constructiva.

\subsection{Descripción de la hipótesis de los periodos constructivos de la iglesia de San Juan de los Reyes}

Se han identificado cinco periodos constructivos para los que se propone una hipótesis de arcos temporales. Las hipótesis están basadas en tres fuentes: el análisis estratigráfico constructivo y el estudio de los ritmos de los mechinales, que proporcionan ambos los datos materiales y una cronología relativa, y el estudio histórico documental, que proporciona los datos de la cronología absoluta.

\section{Periodo 0}

Se trata del periodo identificable con la presencia de la mezquita de los convertidos de Granada, en el mismo solar en que ahora se asienta la iglesia. Se extiende desde la posible fecha de construcción de la mezquita en el siglo XIII hasta la demolición de la misma en enero de 1520. De este periodo, al menos aparentemente, se conserva sólo la estructura del alminar, que se impone con toda su presencia en el exterior de la iglesia y que se refleja en el interior en unos paramentos de tapial fácilmente identificables en las paredes del presbiterio.

\section{Periodo 1}

El periodo 1 comprende la primera fase de construcción de la iglesia, iniciada en 1520 y que todavía seguía en 1538. La iglesia estaba caracterizada, en este periodo, por una estructura muraria muy regular, construida con cajones de argamasa (tierra, cal y cantos rodados) y machones de ladrillo.

\section{Periodo 2}

Se trata del periodo que comprende la segunda fase de construcción, a finales del siglo XVII, y todas las intervenciones de reparación y de mantenimiento realizadas hasta mediados del siglo XIX. Se caracteriza fundamentalmente por la remodelación de la fachada sur, datada según la documentación histórica en 1663, con la construcción de parte del paramento y la inserción de una nueva portada, luego sustituida. La construcción de la pared sur se caracteriza por una fábrica de cajones de argamasa con guijarros de gran tamaño y machones de ladrillo. A este periodo corresponde también una capa de enlucido todavía visible en algunas zonas de la fachada sur de mortero de cal con una capa de acabado superficial de pintura a la cal, con un despiece fingido de sillares. Se trata seguramente de la capa de enlucido que se aplicó después de la renovación de la fachada para uniformar el frente. En el interior de la iglesia en este periodo se abren dos hornacinas, una en lado norte y una en el lado oeste, y se tapia una ventana en el lado sur. Se trata probablemente, también, del momento en que se reviste la estructura del alminar con un paramento de ladrillo.

\section{Periodo 3}

Se trata del periodo en que se somete la iglesia, por parte de los Padres. Redentoristas, a la reforma de finales del siglo XIX. La intervención consiste en una serie de acciones transformadoras enfocadas a la modernización del edificio según el gusto de la época. En la fachada sur, se sustituye la portada existente por una portada neogótica de piedra y se abre el óculo central, intervenciones que se relacionan también con una serie de reparcheos en el paramento de la misma fachada, y la apertura de cuatro ventanas. De la misma manera en la parte superior del mismo frente se tapian las dos ventanas existentes para abrir tres ventanas más pequeñas. En el frente oeste se abren un óculo en la parte superior y un gran ventanal en la inferior, en el centro de la fachada, mientras que en el frente norte simplemente se tapia una ventana. Tras estas intervenciones en el exterior de la iglesia, se enlució la totalidad del edificio con un mortero de cal con un despiece fingido de sillares.

\section{Periodo 4}

El cuarto periodo se caracteriza por la intervención realizada al principio del siglo XX, cuando se abren desde la sacristía hacia el presbiterio dos huecos para emplearlos como tribuna del coro. 


\section{Periodo 5}

Se trata del periodo más reciente, que se caracteriza por una fase importante de transformación de la iglesia y una serie de posteriores intervenciones con el objetivo de frenar la progresiva degradación del edificio. De la intervención de 1929 debida a Torres Balbás, se encuentran fácilmente las huellas de la reconstrucción de la parte superior del muro norte y de la demolición de las bovedillas de la nave sur, sustituidas por un faldón de madera. Desde 1931 hasta nuestros días se han emprendido pequeñas acciones que se evidencian en varias unidades estratigráficas, sin que éstas hayan supuesto un cambio importante para el edificio. 